\title{
CORRIGENDUM
}

\section{Current-induced skyrmion dynamics in constricted geometries}

Junichi Iwasaki, Masahito Mochizuki and Naoto Nagaosa

Nature Nanotechnology 8, 742-747 (2013); published online 8 September 2013; corrected after print 6 January 2014.

In the version of this Article originally published, the authors' competing financial interests were not included. This has now been corrected in the online versions of the Article. 